\title{
REVIEW \\ A reappraisal of the central effects of botulinum neurotoxin type $A$ : by what mechanism?
}

\author{
Matteo Caleo,* Flavia Antonucci, ${ }^{*}$ Laura Restani $\dagger$ and \\ Riccardo Mazzocchio $\ddagger$ \\ *Istituto di Neuroscienze, Consiglio Nazionale delle Ricerche, Pisa, Italy \\ $\uparrow$ Scuola Normale Superiore, Piazza dei Cavalieri, Pisa, Italy \\ $\ddagger$ Sezione di Neurofisiologia Clinica, Dipartimento di Scienze Neurologiche, Neurochirurgiche e del \\ Comportamento, Universita' di Siena, Siena, Italy
}

\begin{abstract}
Botulinum neurotoxin $\mathrm{A}$ (BoNT/A) is a metalloprotease that enters peripheral motor nerve terminals and blocks the release of acetylcholine via the specific cleavage of the synaptosomal-associated protein of $25-\mathrm{kDa}$. Localized injections of BoNT/A are widely employed in clinical neurology to treat several human diseases characterized by muscle hyperactivity. It is generally assumed that the effects of BoNT/A remain localized to the injection site. However, several neurophysiological studies have provided evidence for central
\end{abstract}

effects of BoNT/A, raising the issue of how these actions arise. Here we review these data and discuss the possibility that retrograde axonal transport of catalytically active BoNT/A may explain at least some of its effects at the level of central circuits.

Keywords: botulinum neurotoxins, movement disorders, neuromuscular junction, retrograde axonal transport, synaptic transmission, synaptosomal-associated protein of 25-kDa. J. Neurochem. (2009) 10.1111/j.1471-4159.2009.05887.x
Botulinum neurotoxins are produced by anaerobic bacteria of the genus Clostridium and are the most potent toxins known (Schiavo et al. 2000; Meunier et al. 2002; Montecucco and Molgo 2005). There are seven serotypes of BoNTs, indicated with letters from A to G. Each toxin is composed of a heavy $(\mathrm{H}, 100 \mathrm{kDa})$ and a light chain $(\mathrm{L}, 50 \mathrm{kDa})$ linked by a disulphide bond and non-covalent interactions (Turton et al. 2002). The carboxy-terminus of the heavy chain $\left(\mathrm{H}_{\mathrm{C}}\right)$ binds with extraordinary specificity to nerve terminals. Following receptor-mediated endocytosis and acidification of the endosome, the amino-terminal portion of the heavy chain $\left(\mathrm{H}_{\mathrm{N}}\right)$ translocates the $\mathrm{L}$ chain across the vesicular membrane into the cytosol (Koriazova and Montal 2003). The L chain acts as $\mathrm{Zn}^{2+}$-dependent endopeptidase to cleave essential protein components of the neurotransmitter release machinery, the SNARE (soluble $N$-ethylmaleimide-sensitive factor attachment protein receptor) proteins. This results in disruption of $\mathrm{Ca}^{2+}$-triggered fusion of synaptic vesicles (SVs) with the plasma membrane (Schiavo et al. 2000).

The $\mathrm{L}$ chains of the seven BoNTs are remarkably specific proteases. BoNT/B, /D, /F and /G cleave vesicle-associated membrane protein/synaptobrevin, but each at different sites. BoNT/A and /E cleave synaptosomal-associated protein of 25-kDa (SNAP-25) at two different sites, and BoNT/C attacks both syntaxin and SNAP-25. There are also remarkable differences in the duration of action of the different BoNT serotypes. For example, BoNT/A and BoNT/E cleave the same substrate SNAP-25 (removing respectively 9 and 26 aminoacids from the carboxy terminus), but they cause synaptic blockade with very different properties. Indeed, neuroparalysis triggered by BoNT/E is short-lived, while the blockade induced by BoNT/A lasts for much longer (Eleopra et al. 1998; Keller et al. 1999; Foran et al. 2003b). Factors dictating the short duration of BoNT/E action include limited

Received October 9, 2008; revised manuscript received and accepted January 8, 2009.

Address correspondence and reprint requests to Matteo Caleo, Istituto di Neuroscienze, Consiglio Nazionale delle Ricerche, via G. Moruzzi 1, 56100 Pisa, Italy. E-mail: caleo@in.cnr.it

Abbreviations used: BoNT, botulinum neurotoxin; FGF, fibroblast growth factor; SNAP-25, synaptosomal-associated protein of 25-kDa; SV, synaptic vesicle; TeNT, tetanus neurotoxin. 
stability of this protease within nerve endings and speedy replenishment of BoNT/E-cleaved SNAP-25 (Keller et al. 1999; Adler et al. 2001; Foran et al. 2003b). The long duration of BoNT/A is due (i) to persistent catalytic activity of the protease inside nerve terminals (Keller et al. 1999; Antonucci et al. 2008), and (ii) to the slow replacement of BoNT/A-truncated SNAP-25 (Eleopra et al. 1998; Foran et al. 2003a; Meunier et al. 2003), which interferes with neuroexocytosis by acting as a dominant-negative factor (Foran et al. 2003a; Meunier et al. 2003; Montecucco et al. 2005).

The natural target of BoNTs is represented by the neuromuscular junction, where BoNT poisoning results in flaccid paralysis because of blockade of acetylcholine release. Several experimental studies have shed light on the sequence of events that follow poisoning with BoNT/A (reviewed in Meunier et al. 2002; Foran et al. 2003a). Blockade of exocytosis triggers a remarkable synaptic remodelling in motoneurons, with extensive sprouting and de novo synaptogenesis (de Paiva et al. 1999; Meunier et al. 2002; Morbiato et al. 2007). This sprouting network is responsible for re-establishing functional communication between motoneurons and muscle fibers (de Paiva et al. 1999; Foran et al. 2003a). Importantly, sprouts are eliminated when the original parent terminal recovers its ability to release neurotransmission (de Paiva et al. 1999; Meunier et al. 2003).

The extended duration of BoNT/A effects at the neuromuscular junction is one of the key features that has driven the widespread use of this serotype as a therapeutic agent. Biopharmaceutical preparations of BoNT/A have been used in different neurological diseases (blepharospasm, hemifacial spasm, cervical dystonia, writer's cramp and muscle spasticity) with the aim at weakening contraction in overactive muscles (Jankovic 2004). Importantly, the toxin appears to be taken up preferentially by hyperactive nerve terminals, consistent with the finding that nerve stimulation accelerates BoNT/A poisoning (Hughes and Whaler 1962; Eleopra et al. 1997; Keller et al. 2004; Dong et al. 2006). The peripheral effect of BoNT/A can be monitored by using electrophysiological measures of motor function, such as the maximum direct motor response (Mmax), elicited by peripheral nerve stimulation, which reflects the activation of all motor axons. BoNT/A injections lead to a consistent reduction of Mmax size in parallel with clinical improvement of function. However, this is not always so. It has been observed that the onset and peak of clinical response can be delayed by several days to a week compared to the electrophysiological findings, and the clinical benefit by BoNT/A injection may last much longer than the duration of the neuroparalysis induced by the toxin (Priori et al. 1995; Ziemann et al. 1998; Hardie 2000). On the other hand, patients may request retreatment with BoNT/A in spite of significant residual neuromuscular blockade (Hamjian and Walker 1994). A reduction in the severity of leg spasticity has been reported after BoNT/A injection in spite of little neuromuscular blockade (Mazzocchio et al. 2007). There is also evidence for an improvement of function in muscles acting as antagonists to the injected one (Gracies et al. 2001; Miscio et al. 2004). All these observations are consistent with the suggestion that BoNT/A produces central effects when injected at therapeutic doses in humans (Currà et al. 2004; Abbruzzese and Berardelli 2006).

\section{Evidence for central effects of BoNT/A}

This issue has been addressed by many electrophysiological studies in humans and animal models. Here we briefly review the main findings. Neurophysiological effects of BoNT/A have been found to involve (i) spinal cord circuitry, (ii) brainstem, and (iii) motor cortex.

(i) Spinal cord circuitry. A first set of studies has been conducted in patients with essential tremor or dystonia, two conditions characterized by a dysfunction in the pattern of agonist/antagonist coordinated activity (Rothwell 1995; Ziemann et al. 1998). These patients exhibit reduced presynaptic inhibition of Ia terminals between flexor and extensor forearm muscles (Nakashima et al. 1989; Priori et al. 1995). Presynaptic inhibition is restored to normal levels after injection of BoNT/A in the wrist flexors of these patients (Priori et al. 1995).

A second set of experiments suggests a possible effect of intramuscular BoNT/A on intraspinal recurrent inhibition (Renshaw inhibition). Renshaw cells are glycinergic inhibitory interneurons that receive input from motor axon collaterals and synapse in turn on the somata of motoneurons in a negative-feedback fashion (Alvarez and Fyffe 2007). Evidence for reduced motoneuron input to Renshaw cells after peripheral BoNT/A injection has been obtained from morphological and physiological studies in the rat (Sanna et al. 1993; Gonzalez-Forero et al. 2005; Clowry et al. 2006). However, a cat study showed no effect of intramuscular BoNT/A on the discharge pattern of individual Renshaw cells (Hagenah et al. 1977). Another report demonstrated a decreased recurrent inhibitory activity after BoNT/A muscle injection, resulting in an increase in motoneuron excitability (Wiegand and Wellhoner 1977). These findings were attributed to an action of BoNT/A on the inhibitory synapse between the Renshaw cells and the $\alpha$ motoneurons (Wiegand and Wellhoner 1977). Studies in patients with lower limb spasticity also point to a reduction in the strength of recurrent inhibition following intramuscular BoNT/A (Mazzocchio et al. 2007).

(ii) Brainstem. A series of studies by the group of Delgado-Garcia (Moreno-Lopez et al. 1994, 1997a,b; Pastor et al. 1997) has demonstrated dose-dependent changes in abducens motoneurons following delivery of BoNT/A into the lateral rectus muscle in the cat. Specifically, excitatory 
and inhibitory synaptic transmission to abducens motoneurones is strongly affected several days after BoNT/A injection (Pastor et al. 1997). Moreover, motoneurons show modifications in their firing pattern, as demonstrated by the appearance of an abnormally low discharge rate (MorenoLopez et al. 1994). These changes are accompanied by ultrastructural modifications, such as synaptic stripping at the level of motoneuron somata (Pastor et al. 1997). There is also a reduction in the number of clear vesicles in the terminals impinging onto motoneurons, indicative of an impairment in neuroexocytosis (Pastor et al. 1997). It is worth noting that these changes are dose-dependent (Moreno-Lopez et al. 1994, 1997b). Electrophysiological alterations are modest or absent with doses corresponding to 50-70 mouse units, and become prominent with doses of about 600 mouse units (Moreno-Lopez et al. 1994, 1997b). Human studies revealed no modification of the hyperexcitable brainstem pathways in patients with cranial dystonia (blepharospasm) treated with BoNT/A in the orbicularis oculi muscle (Valls-Sole et al. 1991; Girlanda et al. 1996; Grandas et al. 1998; but see Behari and Raju 1996). In addition, BoNT/A injections for hemifacial spasm and craniocervical dystonia failed to show any effect on brainstem auditory evoked potentials (Behari and Raju 1996; Ce 2000). However, it may well be that the pathways tested by this particular technique are unaffected by BoNT/A central effects. On the other hand, unilateral thyroarytenoid muscle injections of BoNT/A for adductor spasmodic dysphonia led to hypoactivity also in the non-injected muscle, suggesting that speech improvement may in part reflect changes at brainstem level (Bielamowicz and Ludlow 2000).

(iii) Motor cortex. Corticomotor representation of the hand and forearm muscles was shown to be altered in patients with writer's cramp, and these changes could be reversed by BoNT/A injection into the affected muscles (Byrnes et al. 1998). Cortical changes induced by BoNT/A were completely reversible and disappeared at the completion of BoNT/A effects (Byrnes et al. 1998). Contrasting findings have been obtained for the studies concerning motor cortical excitability following BoNT/A. In one study in patients with upper limb dystonia, the clinical benefit of BoNT/A correlated with a return of cortical inhibition to the levels seen in normal subjects (Gilio et al. 2000), but restoration of inhibition has not been confirmed by a later report (Boroojerdi et al. 2003). Finally, abnormally high cortical potentials, evoked by peripheral nerve stimulation, suggestive of increased cortical excitability, were significantly reduced after successful treatment of cervical dystonia with intramuscular BoNT/A (Kanovsky et al. 1998).

Altogether, these data provide evidence that intramuscular BoNT/A produces central effects. The following section addresses the possible mechanisms by which these actions arise.

\section{Possible mechanisms for the central action of BoNT/A}

Botulinum neurotoxin A might affect central circuits by at least three mechanisms, that are schematically summarized in Fig. 1.

(1) BoNT/A-mediated blockade of gamma motor endings, with consequent reduction of spindle afferent input from the injected muscle.

One of the most accredited hypothesis to explain the central physiological effects observed after intramuscular injection of BoNT/A is a concurrent indirect effect on spinal and cortical circuitry, through the action of BoNT/A on the intrafusal, beside the extrafusal, neuromuscular junction (see Currà et al. 2004 for references). Acetylcholine is also the neurotransmitter of $\gamma$-motoneuronal endings in intrafusal muscle fibers, and BoNT/A blockade of intrafusal fibers has been documented in animal studies (Filippi et al. 1993; Rosales et al. 1996). Specifically, at spinal level, lesser muscle afferent input from the wrist flexor (the injected muscle) would decrease the presynaptic gating of the extensor Ia terminals, resulting in a larger presynaptic inhibition of the Ia afferents from the wrist flexors (Priori et al. 1995). At cortical level, reduced muscle afferent input from the injected muscle would lead to a temporary reorganization of the altered sensorimotor interaction (Byrnes et al. 1998), which is a distinctive feature of writer's cramp (Marsden and Sheehy 1990).

(2) Plastic changes following BoNT/A-mediated blockade of neuromuscular transmission.

Theoretically, two sites of plasticity can be envisaged following blockade of neuromuscular transmission: one regarding the motoneuron itself, and the other concerning the target muscle. Indeed, there is evidence for rearrangements at both sites.

The functional and structural properties of motoneurons are known to vary following blockade of neuromuscular transmission with BoNT/A. At the functional level, changes in rheobase current, input resistance, time constant, and duration of the after-hyperpolarization are observed in spinal motoneurons of cats treated with BoNT/A into the medial gastrocnemius muscle (Pinter et al. 1991). Metabolic changes have been observed in rat hypoglossal motoneurons after intramuscular BoNT/A (Watson 1969). Blockade of synaptic transmission triggers changes in the expression of numerous genes in spinal motoneurons (Jung et al. 1997). In addition, significant reductions in the density of gephyrin, a glycine receptor clustering protein, on the membrane of cat abducens motoneurons have been reported after BoNT/A injection in their target muscle (Moreno-Lopez et al. 1998). This confirms alterations in synaptic input to motoneurons poisoned with BoNT/A.

A second site of significant plasticity is represented by the injected muscle. To our knowledge, not much emphasis has 


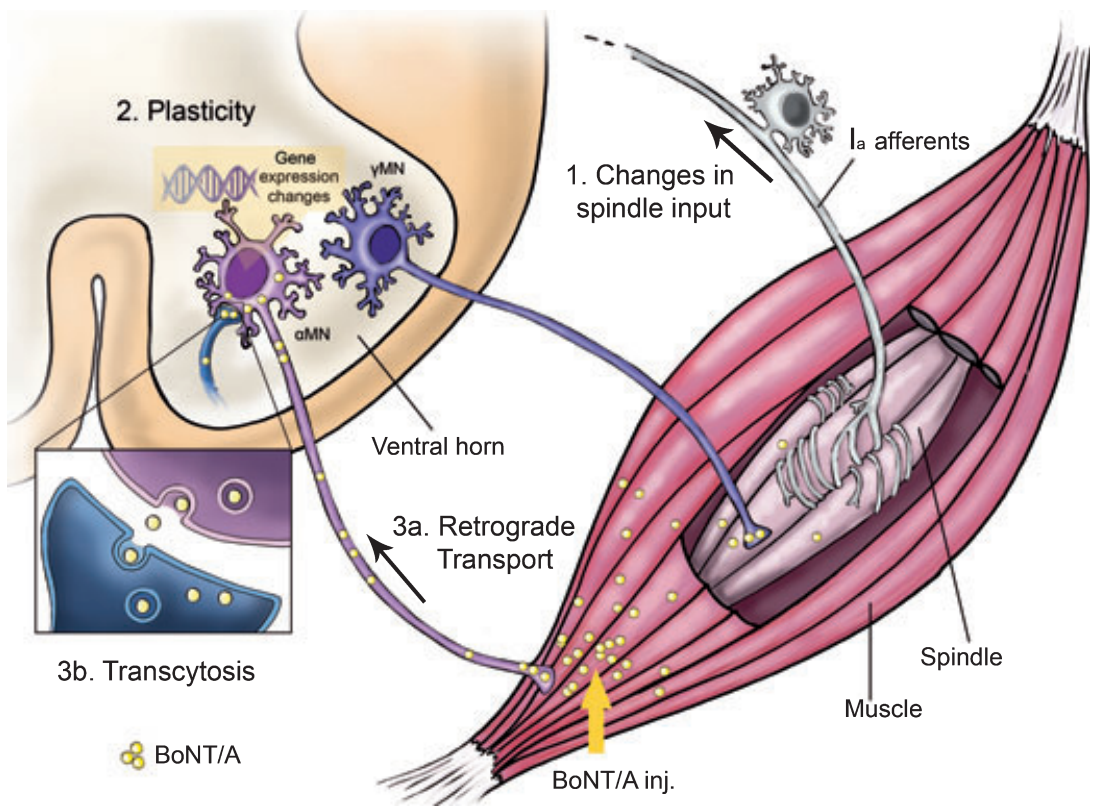

Fig. 1 Sketch of the extra- and intra-fusal muscle fibers with their afferent and efferent innervation and possible mechanisms for the central action of BoNT/A. (1) Presynaptic blockade of the neuromuscular connection between $\gamma$-motoneuronal endings and intrafusal muscle fibers with consequent reduction of input from la afferents. This may cause changes in the excitability of spinal pathways mediating presynaptic inhibition of la afferents to antagonist muscles, as well as transitory changes in motor maps at cortical level. (2) Pre- synaptic blockade of the neuromuscular connection between $\alpha$ motoneuronal endings and extrafusal muscle fibers may cause plastic changes in the motoneuron (e.g. changes in gene expression). (3) Retrograde transport along the motor axon to the motoneuron (3a) and transcytosis to afferent synapses (3b). Such possibility allows for direct central effects of BoNT/A. $\alpha \mathrm{MN}, \alpha$-motoneuron; $\gamma \mathrm{MN}, \gamma$-motoneuron. been given to the role of muscle fiber type in the long term changes following BoNT/A injection. Muscles contain a characteristic mixture of slow fibres, innervated by tonically firing motoneurones and generating relatively little force but easily recruited and resistant to fatigue, and fast fibres, innervated by phasically active motoneurones which generate larger amounts of force (Henneman and Mendell 1991). Each fibre type expresses characteristic slow or fast myosin heavy chain with differing metabolic properties appropriate to the functional class of the muscle fibre (Pette and Staron 2000). Motoneurons innervating slow muscle fibres fire many more action potentials on a daily basis than do motoneurons that innervate fast muscle fibres (Hennig and Lomo 1985). Thus, nerve paralysis will eliminate proportionately more evoked acetylcholine release among slow than fast motoneurons. This may explain the differential effects of BoNT/A on motoneuron electrical properties according to fibre type (Pinter et al. 1991) and, perhaps, why the terminals of slow motoneurones sprout more vigorously than those of fast motoneurons after BoNT/A (Duchen 1970; Tonge 1974; Brown 1984; Frey et al. 2000). Muscle fibre phenotype is largely governed by motor axon activity patterns (Navarrette and Vrbova 1993), and so slow motor axons could take over fast fibres by collateral sprouting following blockade of neuromuscular transmission by BoNT/A. Indeed, there is evidence for a change in muscle fibres' phenotype, as estimated by an increased expression of slow myosin heavy chain, in BoNT/A-treated muscles (Inagi et al. 1999; Frey et al. 2000; Dodd et al. 2005; Clowry et al. 2006). These findings may explain the clinical observation that BoNT/A injections occasionally leave the injected muscle disproportionately weak: if slow motor axons take over fast fibres, the result will be less tension and a weaker muscle. This notion may have important functional consequences for the treatment of the spastic muscle, which shows differential and time-dependent changes in its contractile properties (Hufschmidt and Mauritz 1985; Gracies 2005).

(3) Retrograde transport and transcytosis of BoNT/A following uptake at the neuromuscular junction.

Botulinum neurotoxin A applied in the periphery could directly affect central circuits via retrograde transport and trancytosis (Antonucci et al. 2008). The notion that BoNT/A could reach the central nervous system by retrograde axonal transport was initially supported by experiments with radiolabelled BoNT/A. It was found that the toxin is transferred to the ventral roots and adjacent spinal cord segments upon intramuscular injection in the cat (Habermann 1974; Wiegand et al. 1976). Black and Dolly (1986) 
observed radiolabelled BoNT/A within the axoplasm of myelinated axons after peripheral injection of the toxin in mice, suggesting a retrograde intra-axonal transfer of the toxin. However, it has been argued that the retrograde axonal transport was so slow that the applied BoNT/A was likely to be inactivated before it reached the cell soma (Black and Dolly 1986; Dressler and Adib Saberi 2005). Contrary to these arguments, it has been recently shown that BoNT/A is also capable of long-distance effects after application at the neuromuscular junction. Whilst most of the BoNT/A effects remained restricted to the injection site, there were signs of toxin activity also in distant synapses. Specifically, cleavage of the BoNT/A substrate SNAP-25 was detected in the rat facial nucleus following delivery of BoNT/A to the whisker pad (Antonucci et al. 2008).

An important issue is whether the central effects of BoNT/ A depend on dosage. First, it is important to point out that several neurophysiological studies in patients indicate central actions of BoNT/A when injected intramuscularly at therapeutic doses (see above). Second, evidence from animal experiments has provided proof-of-principle for a direct central effect of BoNT/A via retrograde transport, especially at high doses (Wiegand et al. 1976; Moreno-Lopez et al. 1994; Pastor et al. 1997; Antonucci et al. 2008). For example, Antonucci et al. (2008) reported retrograde transport after injecting about 5-6 BoNT/A units into the whisker pad of adult rats (i.e., 15 units $/ \mathrm{kg}$ body weight, taking into consideration that an adult rat weighs $350-400 \mathrm{~g}$ ). Total dosage of up to 800 units can be employed for the treatment of dystonia and spasticity in patients (e.g. Garner et al. 1993; Wohlfarth et al. 2001; Jankovic 2004). Considering an average body weight of $70 \mathrm{~kg}$, this leads to a maximum dose of about 11.5 units $/ \mathrm{kg}$ in humans, which is slightly lower than the amount used in Antonucci et al. (2008). However, a direct comparison of animal and human data should be regarded with caution, as retrograde transport of BoNT/A is likely to depend not only on the total amount of injected toxin, but also on type and size of the muscle, density of innervation, and levels of expression of toxin receptors in the specific pathways under consideration.

\section{Evidence for retrograde transport and transcytosis of catalytically active BoNT/A}

Experiments in the visual system have provided conclusive evidence that at least a fraction of the injected BoNT/A undergoes retrograde axonal transport in neurons and is then transcytosed in a catalytically active form to afferent synapses (Antonucci et al. 2008). These studies consisted in injections of BoNT/A into the rat superior colliculus, a midbrain area that receives a massive, unidirectional projection from ganglion cells in the retina (see Fig. 2a). Three days (but not one day) after injection, BoNT/A-truncated SNAP-25 could be detected in retinal synapses impinging onto ganglion cells (Antonucci et al. 2008; Fig. 2a). Importantly, cleavage of SNAP-25 was only apparent in neuronal populations directly connected to the superior colliculus, and appearance of truncated SNAP-25 in the retina was blocked by the axonal transport blocker colchicine, thus ruling out a systemic spread (Antonucci et al. 2008). Appearance of BoNT/A-altered SNAP-25 in retinal synapses suggested retrograde axonal transport and transcytosis, but it was at least theoretically possible that the cleaved SNAP-25 circulated, rather than the intact toxin. To prove retrograde transfer and transcytosis of catalytically active BoNT/A, retinal ganglion cell axons were cut (to prevent further transport from the colliculus) and BoNT/E was injected intraocularly (Fig. 2b). It is well known that BoNT/A removes the last nine residues from the $\mathrm{C}$-terminus of SNAP-25, while BoNT/E cleaves a larger 26 residue fragment from the same region of SNAP-25; thus, the intraocular injection of BoNT/E removed BoNT/A-truncated SNAP-25 from retinal synapses (Fig. 2b; Lawrence et al. 1997; Keller et al. 1999; Adler et al. 2001). This loss of BoNT/A-truncated SNAP-25 should be permanent if the cleaved substrate is transported, as there is no way of generating new BoNT/A-altered SNAP-25 in the retina. Conversely, if active BoNT/A is transported from the colliculus, its action would reappear when BoNT/E effects are extinguished (Keller et al. 1999; Adler et al. 2001). Indeed, BoNT/A-mediated cleavage reappeared at the completion of BoNT/E effects (Fig. 2b). The return of BoNT/Atruncated SNAP-25 demonstrates that BoNT/A is retrogradely transported from the optic tectum and persists in a catalytically active form in retinal terminals.

\section{BoNT/A retrograde transport: possible mechanisms and implications}

To reach distant synapses, BoNT/A has to be loaded onto retrogradely moving organelles, escape degradation in the cell soma, be released at postsynaptic sites, undergo a second cycle of uptake, and finally be released into the cytosol to exert its proteolytic activity. This pathway is typical of tetanus neurotoxin (TeNT), another member of the clostridial family of toxins (Lalli et al. 2003; Rind et al. 2005). TeNT is internalized in neurons via a process of endocytosis that requires the activity of dynamin and other classical clathrin endocytic adaptors, and is then sorted towards the retrograde transport route (Deinhardt et al. 2006a,b). It will be important in future studies to determine if there is some overlap in the intracellular trafficking events that involve TeNT and BoNT/A. Another issue is whether there are differences in trafficking between BoNT/A and other BoNT serotypes. Ultrastructural analysis has revealed radiolabelled BoNT/B within myelinated axons after peripheral injection of the toxin, indicative of retrograde axonal transport (Black and Dolly 1986). Other experiments have failed to detect 
(a)

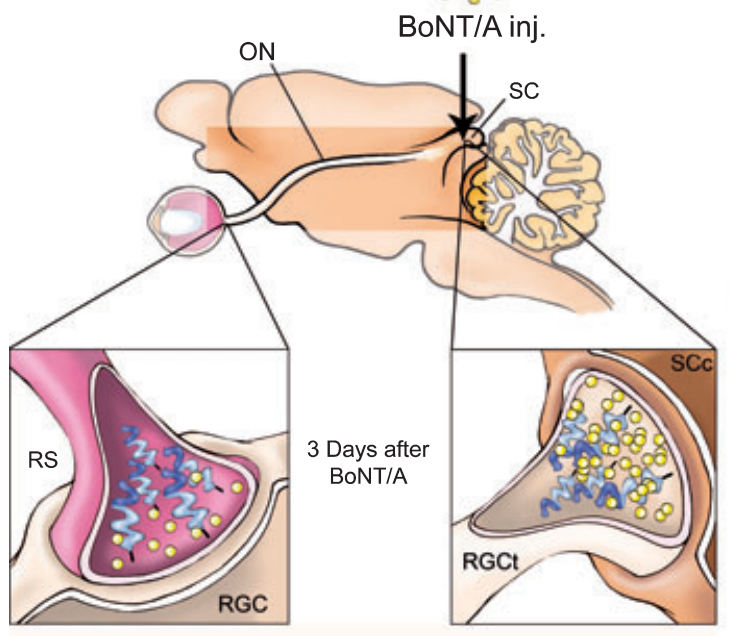

(b)
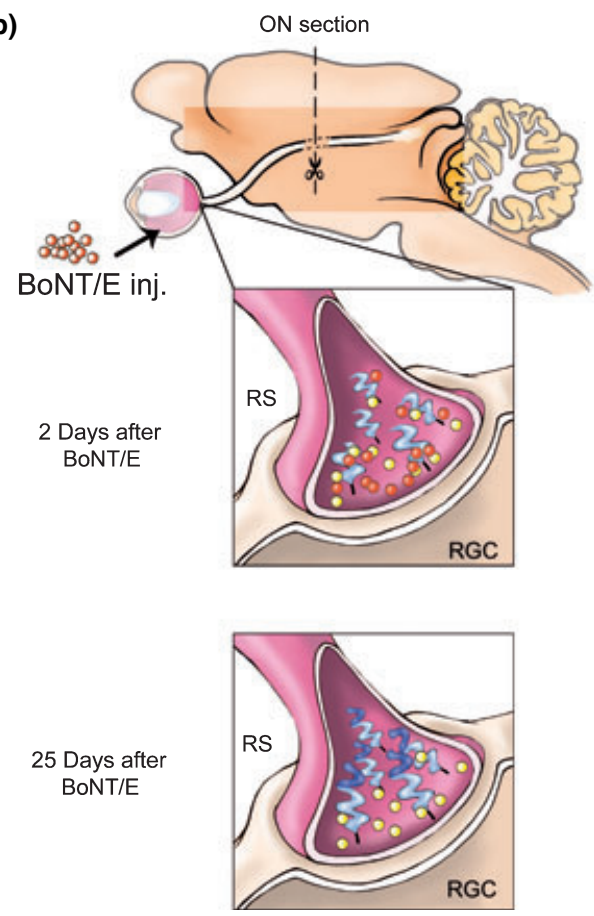

retrograde transport and transcytosis of BoNT/E, at least in hippocampal neurons (Antonucci et al. 2008).

The mechanisms involved in retrograde transport and transcytosis of BoNT/A remain to be defined. Only some hypotheses can be drawn at this stage. Entry of BoNT/A into neurons is known to be mediated via SV recycling (Verderio et al. 2006). Specifically, BoNT/A interacts with the luminal domain of SV2 (Dong et al. 2006; Mahrhold et al. 2006). Acidification of the recycled vesicle should then lead to release of the L chain of BoNT/A, thus preventing retrograde axonal transport. Interestingly, however, it is known that BoNT/A-containing vesicles acidify quite slowly as compared to BoNT/E-containing endosomes (Keller et al. 2004;

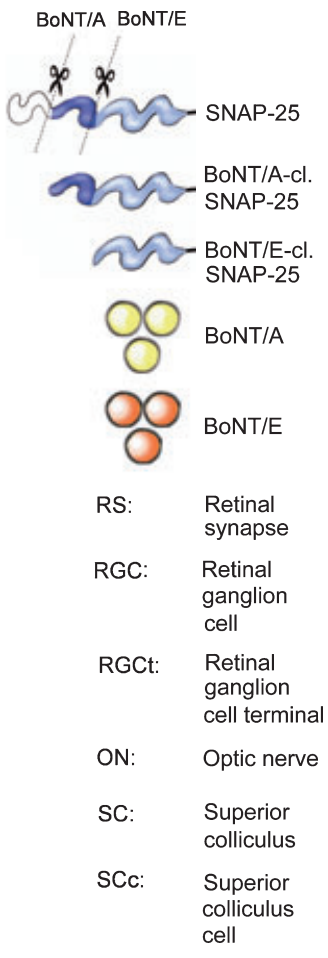

Fig. 2 Experimental design for the demonstration of retrograde transport of active BoNT/A in the visual system. (a) BoNT/A is injected into the superior colliculus (SC) of rats, where it exerts most of its proteolytic activity. Three days after toxin delivery, significant amounts of BoNT/A-truncated SNAP-25 are also detectable in retinal terminals impinging onto retinal ganglion cells. (b) To demonstrate retrograde transfer and transcytosis of active BoNT/A, retinal ganglion cell axons are cut (to prevent additional transport from the colliculus) and BoNT/E is injected intraocularly (top panel). BoNT/E removes BoNT/A-truncated SNAP25 from retinal synapses and generates BoNT/E-altered SNAP-25 (middle panel). However, BoNT/A-mediated cleavage reappears at the completion of BoNT/E effects (25 days post-BoNT/E, lower panel), demonstrating persistent catalytic activity of BoNT/A within the retina.

Wang et al. 2008). This delayed translocation of BoNT/A might allow the BoNT/A cargoes to be loaded onto the axonal transport machinery. Indeed, there is evidence that SVs shuttle between synaptic terminals and display considerable motility in axons (Darcy et al. 2006).

Botulinum neurotoxin A has also been reported to bind the fibroblast growth factor (FGF) receptor 3 (Fernandez-Salas et al. 2008). Thus, another possibility is that BoNT/A is trafficked retrogradely following interaction with the FGF receptor 3, as FGF is well known to undergo axonal transport in neurons (Mufson et al. 1999).

One crucial determinant of the long-distance effects of BoNT/A is the type(s) of synapses that are affected in remote 
areas following retrograde transport of the toxin. For example, tetanus toxin is retrogradely transported to spinal cord motoneurons and selectively transcytosed into inhibitory cells, thus resulting in spastic paralysis. Some specificity in transcytosis appear to exist also for BoNT/A. Indeed, in the study of BoNT/A trafficking cited above (Antonucci et al. 2008), BoNT/A effects were mainly observed in cholinergic synapses of the retina following injection of the toxin into the superior colliculus. Interestingly, retinal cholinergic terminals express high levels of SV2C (Wang et al. 2003; Antonucci et al. 2008), the SV2 isoform that exhibits the most robust BoNT/A binding activity (Dong et al. 2006; Mahrhold et al. 2006). Thus, retinal cholinergic cells might take up BoNT/A more efficiently because of SV2C receptor expression.

The central synapses that are targeted by BoNT/A after retrograde transport in motoneurons remain to be determined. It is conceivable that such direct central actions may act synergistically with the peripheral blockade (for example in the case of the silencing of an excitatory input to the motoneuron, that would reinforce the peripheral effect). In other cases, additional circuits may be affected. Indeed, in vitro and in vivo studies demonstrate that BoNT/A has the potential to interfere with release of several neurotransmitters from central neurons (Ashton and Dolly 1988; Luvisetto et al. 2003, 2004; Bozzi et al. 2006; Verderio et al. 2007). Wiegand and Wellhoner (1977) postulated a direct action of intramuscular BoNT/A on inhibitory connections between Renshaw cells and alpha-motoneurons. Cholinergic inputs from motor axon collaterals to Renshaw cells might be affected as well (Sanna et al. 1993; Gonzalez-Forero et al. 2005; Clowry et al. 2006; but see Hagenah et al. 1977). The physiological data in cats injected with BoNT/A into the lateral rectus muscle are consistent with a specific blocking effect of the toxin on vestibular and reticular afferents onto abducens motoneurons (Moreno-Lopez et al. 1994, 1997b).

\section{Concluding remarks}

From a clinical point of view, BoNT/A is an excellent drug for the treatment of neuromuscular pathologies, such as dystonia and spasticity. In both pathologies, treatment with BoNT/A has advantageously replaced surgical procedures. The range of clinical applications of BoNT/A is continuously increasing to include treatment of a variety of ophtalmological, gastrointestinal, urological, orthopaedic, dermatological, secretory, painful, and cosmetic disorders. Despite this widespread use, relatively little is known on BoNT/A intracellular trafficking and potential central effects. It is therefore important to fully characterize the spectrum of actions of this neurotoxin. There is substantial evidence that intramuscular injection of BoNT/ A results in central nervous system effects. These findings have been usually ascribed to plastic rearrangements subsequent to the peripheral blockade. The finding of a retrograde transport of catalytically active BoNT/A suggests that BoNT/A may also have direct central effects, especially at high doses. It is not yet clear whether these central effects actually contribute to the therapeutic efficacy of BoNT/A. A more detailed understanding of the central actions of BoNT/A will provide valuable information for present and future uses of this neurotoxin in clinical practice.

\section{Acknowledgements}

We are indebted to Dr. Matilde Marchi (Menerva Srl, Pisa) for drawing the Figures. This work was supported by a grant (P94) from the International Institute for Research in Paraplegia, Zurich, Switzerland, and by FIRB Project CHEM-PROFARMA-NET.

\section{References}

Abbruzzese G. and Berardelli A. (2006) Neurophysiological effects of botulinum toxin type A. Neurotox. Res. 9, 109-114.

Adler M., Keller J. E., Sheridan R. E. and Deshpande S. S. (2001) Persistence of botulinum neurotoxin A demonstrated by sequential administration of serotypes $\mathrm{A}$ and $\mathrm{E}$ in rat EDL muscle. Toxicon 39, 233-243.

Alvarez F. J. and Fyffe R. E. (2007) The continuing case for the Renshaw cell. J. Physiol. 584, 31-45.

Antonucci F., Rossi C., Gianfranceschi L., Rossetto O. and Caleo M. (2008) Long-distance retrograde effects of botulinum neurotoxin A. J. Neurosci. 28, 3689-3696.

Ashton A. C. and Dolly J. O. (1988) Characterization of the inhibitory action of botulinum neurotoxin type A on the release of several transmitters from rat cerebrocortical synaptosomes. J. Neurochem. 50, 1808-1816.

Behari M. and Raju G. B. (1996) Electrophysiological studies in patients with blepharospasm before and after botulinum toxin A therapy. J. Neurol. Sci. 135, 74-77.

Bielamowicz S. and Ludlow C. L. (2000) Effects of botulinum toxin on pathophysiology in spasmodic dysphonia. Ann. Otol. Rhinol. Laryngol. 109, 194-203.

Black J. D. and Dolly J. O. (1986) Interaction of 125I-labeled botulinum neurotoxins with nerve terminals. I. Ultrastructural autoradiographic localization and quantitation of distinct membrane acceptors for types A and B on motor nerves. J. Cell Biol. 103, 521-534.

Boroojerdi B., Cohen L. G. and Hallett M. (2003) Effects of botulinum toxin on motor system excitability in patients with writer's cramp. Neurology 61, 1546-1550.

Bozzi Y., Costantin L., Antonucci F. and Caleo M. (2006) Action of botulinum neurotoxins in the central nervous system: antiepileptic effects. Neurotox. Res. 9, 197-203.

Brown M. (1984) Sprouting of motor nerves in adult muscles: a recapitulation of ontogeny. Trends Neurosci. 7, 10-14.

Byrnes M. L., Thickbroom G. W., Wilson S. A., Sacco P., Shipman J. M., Stell R. and Mastaglia F. L. (1998) The corticomotor representation of upper limb muscles in writer's cramp and changes following botulinum toxin injection. Brain 121, 977-988.

Ce P. (2000) Central effects of botulinum toxin: study of brainstem auditory evoked potentials. Eur. J. Neurol. 7, 747.

Clowry G. J., Walker L. and Davies P. (2006) The effects of botulinum neurotoxin A induced muscle paresis during a critical period upon muscle and spinal cord development in the rat. Exp. Neurol. 202, 456-469. 
Currà A., Trompetto C., Abbruzzese G. and Berardelli A. (2004) Central effects of botulinum toxin type A: evidence and supposition. Mov. Disord. 19(Suppl. 8), S60-S64.

Darcy K. J., Staras K., Collinson L. M. and Goda Y. (2006) Constitutive sharing of recycling synaptic vesicles between presynaptic boutons. Nat. Neurosci. 9, 315-321.

Deinhardt K., Berninghausen O., Willison H. J., Hopkins C. R. and Schiavo G. (2006a) Tetanus toxin is internalized by a sequential clathrin-dependent mechanism initiated within lipid microdomains and independent of epsin1. J. Cell Biol. 174, 459-471.

Deinhardt K., Salinas S., Verastegui C., Watson R., Worth D., Hanrahan S., Bucci C. and Schiavo G. (2006b) Rab5 and Rab7 control endocytic sorting along the axonal retrograde transport pathway. Neuron 52, 293-305.

Dodd S. L., Selsby J., Payne A., Judge A. and Dott C. (2005) Botulinum neurotoxin type A causes shifts in myosin heavy chain composition in muscle. Toxicon 46, 196-203.

Dong M., Yeh F., Tepp W. H., Dean C., Johnson E. A., Janz R. and Chapman E. R. (2006) SV2 is the protein receptor for botulinum neurotoxin A. Science 312, 592-596.

Dressler D. and Adib Saberi F. (2005) Botulinum toxin: mechanisms of action. Eur. Neurol. 53, 3-9.

Duchen L. W. (1970) Changes in motor innervation and cholinesterase localization induced by botulinum toxin in skeletal muscle of the mouse: differences between fast and slow muscles. J. Neurol. Neurosurg. Psychiatry 33, 40-54.

Eleopra R., Tugnoli V. and De Grandis D. (1997) The variability in the clinical effect induced by botulinum toxin type A: the role of muscle activity in humans. Mov. Disord. 12, 89-94.

Eleopra R., Tugnoli V., Rossetto O., De Grandis D. and Montecucco C. (1998) Different time courses of recovery after poisoning with botulinum neurotoxin serotypes A and E in humans. Neurosci. Lett. 256, 135-138

Fernandez-Salas E., Garay P., Jacky B., Dupuy J., Wang J., Nelson J., Raymond C. S. and Aoki K. R. (2008) Identification of the fibroblast growth factor receptor FGFR3 as a component of the receptor complex for Botulinum Neurotoxin Type A. Toxicon 5(Suppl. 1), 3.

Filippi G. M., Errico P., Santarelli R., Bagolini B. and Manni E. (1993) Botulinum A toxin effects on rat jaw muscle spindles. Acta Otolaryngol. 113, 400-404.

Foran P. G., Davletov B. and Meunier F. A. (2003a) Getting muscles moving again after botulinum toxin: novel therapeutic challenges. Trends Mol. Med. 9, 291-299.

Foran P. G., Mohammed N., Lisk G. O., Nagwaney S., Lawrence G. W., Johnson E., Smith L., Aoki K. R. and Dolly J. O. (2003b) Evaluation of the therapeutic usefulness of botulinum neurotoxin $\mathrm{B}, \mathrm{C} 1$, $\mathrm{E}$, and $\mathrm{F}$ compared with the long lasting type A. Basis for distinct durations of inhibition of exocytosis in central neurons. J. Biol. Chem. 278, 1363-1371.

Frey D., Schneider C., Xu L., Borg J., Spooren W. and Caroni P. (2000) Early and selective loss of neuromuscular synapse subtypes with low sprouting competence in motoneuron diseases. J. Neurosci. 20, 2534-2542.

Garner C. G., Straube A., Witt T. N., Gasser T. and Oertel W. H. (1993) Time course of distant effects of local injections of botulinum toxin. Mov. Disord. 8, 33-37.

Gilio F., Curra A., Lorenzano C., Modugno N., Manfredi M. and Berardelli A. (2000) Effects of botulinum toxin type A on intracortical inhibition in patients with dystonia. Ann. Neurol. 48, 2026.

Girlanda P., Quartarone A., Sinicropi S., Nicolosi C. and Messina C. (1996) Unilateral injection of botulinum toxin in blepharospasm: single fiber electromyography and blink reflex study. Mov. Disord. 11, 27-31.

Gonzalez-Forero D., Pastor A. M., Geiman E. J., Benitez-Temino B. and Alvarez F. J. (2005) Regulation of gephyrin cluster size and inhibitory synaptic currents on Renshaw cells by motor axon excitatory inputs. J. Neurosci. 25, 417-429.

Gracies J. M. (2005) Pathophysiology of spastic paresis. I: paresis and soft tissue changes. Muscle Nerve 31, 535-551.

Gracies J., Weisz D., Yang B., Flanagan S. and Simpson D. (2001) Evidence for increased antagonist strength and movement speed following botulinum toxin injections in spasticity. Neurology 56, A3.

Grandas F., Traba A., Alonso F. and Esteban A. (1998) Blink reflex recovery cycle in patients with blepharospasm unilaterally treated with botulinum toxin. Clin. Neuropharmacol. 21, $307-$ 311 .

Habermann E. (1974) 125I-labeled neurotoxin from Clostridium botulinum A: preparation, binding to synaptosomes and ascent to the spinal cord. Naunyn Schmiedebergs Arch. Pharmacol. 281, 47-56.

Hagenah R., Benecke R. and Wiegand H. (1977) Effects of type A botulinum toxin on the cholinergic transmission at spinal Renshaw cells and on the inhibitory action at Ia inhibitory interneurones. Naunyn Schmiedebergs Arch. Pharmacol. 299, 267-272.

Hamjian J. A. and Walker F. O. (1994) Serial neurophysiological studies of intramuscular botulinum-A toxin in humans. Muscle Nerve 17 1385-1392.

Hardie R. J. (2000) Botulinum toxin in muscle spasticity. J. Neurol. Neurosurg. Psychiatry 68, 689-690.

Henneman E. and Mendell L. M. (1991) Functional Organisation of the Motoneuron Pool and its Inputs. Williams and Wilkins, Bethesda, MD.

Hennig R. and Lomo T. (1985) Firing patterns of motor units in normal rats. Nature 314, 164-166.

Hufschmidt A. and Mauritz K. H. (1985) Chronic transformation of muscle in spasticity: a peripheral contribution to increased tone. J. Neurol. Neurosurg. Psychiatry 48, 676-685.

Hughes R. and Whaler B. C. (1962) Influence of nerve-ending activity and of drugs on the rate of paralysis of rat diaphragm preparations by $\mathrm{Cl}$. botulinum type A toxin. J. Physiol. 160, 221-233.

Inagi K., Connor N. P., Schultz E., Ford C. N., Cook C. H. and Heisey D. M. (1999) Muscle fiber-type changes induced by botulinum toxin injection in the rat larynx. Otolaryngol. Head Neck Surg. 120, 876-883.

Jankovic J. (2004) Botulinum toxin in clinical practice. J. Neurol. Neurosurg. Psychiatry 75, 951-957.

Jung H. H., Lauterburg T. and Burgunder J. M. (1997) Expression of neurotransmitter genes in rat spinal motoneurons after chemodenervation with botulinum toxin. Neuroscience 78, 469-479.

Kanovsky P., Streitova H., Dufek J., Znojil V., Daniel P. and Rektor I. (1998) Change in lateralization of the P22/N30 cortical component of median nerve somatosensory evoked potentials in patients with cervical dystonia after successful treatment with botulinum toxin A. Mov. Disord. 13, 108-117.

Keller J. E., Neale E. A., Oyler G. and Adler M. (1999) Persistence of botulinum neurotoxin action in cultured spinal cord cells. FEBS Lett. 456, 137-142.

Keller J. E., Cai F. and Neale E. A. (2004) Uptake of botulinum neurotoxin into cultured neurons. Biochemistry 43, 526-532.

Koriazova L. K. and Montal M. (2003) Translocation of botulinum neurotoxin light chain protease through the heavy chain channel. Nat. Struct. Biol. 10, 13-18. 
Lalli G., Bohnert S., Deinhardt K., Verastegui C. and Schiavo G. (2003) The journey of tetanus and botulinum neurotoxins in neurons. Trends Microbiol. 11, 431-437.

Lawrence G. W., Foran P., Mohammed N., DasGupta B. R. and Dolly J. O. (1997) Importance of two adjacent C-terminal sequences of SNAP-25 in exocytosis from intact and permeabilized chromaffin cells revealed by inhibition with botulinum neurotoxins $\mathrm{A}$ and $\mathrm{E}$. Biochemistry 36, 3061-3067.

Luvisetto S., Rossetto O., Montecucco C. and Pavone F. (2003) Toxicity of botulinum neurotoxins in central nervous system of mice. Toxicon 41, 475-481.

Luvisetto S., Marinelli S., Rossetto O., Montecucco C. and Pavone F. (2004) Central injection of botulinum neurotoxins: behavioural effects in mice. Behav. Pharmacol. 15, 233-240.

Mahrhold S., Rummel A., Bigalke H., Davletov B. and Binz T. (2006) The synaptic vesicle protein $2 \mathrm{C}$ mediates the uptake of botulinum neurotoxin A into phrenic nerves. FEBS Lett. 580, 2011-2014.

Marsden C. D. and Sheehy M. P. (1990) Writer's cramp. Trends Neurosci. 13, 148-153.

Mazzocchio R., Spidalieri R., Dominici F., Popa T., Hallett M. and Rossi A. (2007) Putative central effects of botulinum toxin, possibly mediated by changes in Renshaw cell activity, following intramuscular injection in humans. Mov. Disord. 22, S122.

Meunier F. A., Schiavo G. and Molgo J. (2002) Botulinum neurotoxins: from paralysis to recovery of functional neuromuscular transmission. J. Physiol. Paris 96, 105-113.

Meunier F. A., Lisk G., Sesardic D. and Dolly J. O. (2003) Dynamics of motor nerve terminal remodeling unveiled using SNAREcleaving botulinum toxins: the extent and duration are dictated by the sites of SNAP-25 truncation. Mol. Cell. Neurosci. 22, 454466.

Miscio G., Del Conte C., Pianca D., Colombo R., Panizza M., Schieppati M. and Pisano F. (2004) Botulinum toxin in post-stroke patients: stiffness modifications and clinical implications. J. Neurol. 251, 189-196.

Montecucco C. and Molgo J. (2005) Botulinal neurotoxins: revival of an old killer. Curr. Opin. Pharmacol. 5, 274-279.

Montecucco C., Schiavo G. and Pantano S. (2005) SNARE complexes and neuroexocytosis: how many, how close? Trends Biochem. Sci. 30, 367-372.

Morbiato L., Carli L., Johnson E. A., Montecucco C., Molgo J. and Rossetto O. (2007) Neuromuscular paralysis and recovery in mice injected with botulinum neurotoxins A and C. Eur. J. Neurosci. 25, 2697-2704

Moreno-Lopez B., de la Cruz R. R., Pastor A. M. and Delgado-Garcia J. M. (1994) Botulinum neurotoxin alters the discharge characteristics of abducens motoneurons in the alert cat. J. Neurophysiol. 72, 2041-2044

Moreno-Lopez B., de la Cruz R. R., Pastor A. M. and Delgado-Garcia J. M. (1997a) Effects of botulinum neurotoxin type A on abducens motoneurons in the cat: alterations of the discharge pattern. Neuroscience $\mathbf{8 1}, 437-455$.

Moreno-Lopez B., Pastor A. M., de la Cruz R. R. and Delgado-Garcia J. M. (1997b) Dose-dependent, central effects of botulinum neurotoxin type A: a pilot study in the alert behaving cat. Neurology 48, 456-464.

Moreno-Lopez B., de la Cruz R. R., Pastor A. M., Delgado-Garcia J. M. and Alvarez F. J. (1998) Effects of botulinum neurotoxin type A on the expression of gephyrin in cat abducens motoneurons. J. Comp. Neurol. 400, 1-17.

Mufson E. J., Kroin J. S., Sendera T. J. and Sobreviela T. (1999) Distribution and retrograde transport of trophic factors in the central nervous system: functional implications for the treatment of neurodegenerative diseases. Prog. Neurobiol. 57, 451-484.
Nakashima K., Rothwell J. C., Day B. L., Thompson P. D., Shannon K. and Marsden C. D. (1989) Reciprocal inhibition between forearm muscles in patients with writer's cramp and other occupational cramps, symptomatic hemidystonia and hemiparesis due to stroke. Brain 112, 681-697.

Navarrette R. and Vrbova G. (1993) Activity-dependent interactions between motoneurones and muscles: their role in the development of the motor unit. Prog. Neurobiol. 41, 93-124.

de Paiva A., Meunier F. A., Molgo J., Aoki K. R. and Dolly J. O. (1999) Functional repair of motor endplates after botulinum neurotoxin type A poisoning: biphasic switch of synaptic activity between nerve sprouts and their parent terminals. Proc. Natl Acad. Sci. USA 96, 3200-3205.

Pastor A. M., Moreno-Lopez B., De La Cruz R. R. and Delgado-Garcia J. M. (1997) Effects of botulinum neurotoxin type A on abducens motoneurons in the cat: ultrastructural and synaptic alterations. Neuroscience 81, 457-478.

Pette D. and Staron R. S. (2000) Myosin isoforms, muscle fiber types, and transitions. Microsc. Res. Tech. 50, 500-509.

Pinter M. J., Vanden Noven S., Muccio D. and Wallace N. (1991) Axotomy-like changes in cat motoneuron electrical properties elicited by botulinum toxin depend on the complete elimination of neuromuscular transmission. J. Neurosci. 11, 657-666.

Priori A., Berardelli A., Mercuri B. and Manfredi M. (1995) Physiological effects produced by botulinum toxin treatment of upper limb dystonia. Changes in reciprocal inhibition between forearm muscles. Brain 118(Pt 3), 801-807.

Rind H. B., Butowt R. and von Bartheld C. S. (2005) Synaptic targeting of retrogradely transported trophic factors in motoneurons: comparison of glial cell line-derived neurotrophic factor, brain-derived neurotrophic factor, and cardiotrophin-1 with tetanus toxin. J. Neurosci. 25, 539-549.

Rosales R. L., Arimura K., Takenaga S. and Osame M. (1996) Extrafusal and intrafusal muscle effects in experimental botulinum toxin-A injection. Muscle Nerve 19, 488-496.

Rothwell J. C. (1995) Pathophysiology of Essential Tremor. Marcel Dekker, New York.

Sanna P. P., Celio M. R., Bloom F. E. and Rende M. (1993) Presumptive Renshaw cells contain decreased calbindin during recovery from sciatic nerve lesions. Proc. Natl Acad. Sci. USA 90, 3048-3052.

Schiavo G., Matteoli M. and Montecucco C. (2000) Neurotoxins affecting neuroexocytosis. Physiol. Rev. 80, 717-766.

Tonge D. A. (1974) Chronic effects of botulinum toxin on neuromuscular transmission and sensitivity to acetylcholine in slow and fast skeletal muscle of the mouse. J. Physiol. 241, 127-139.

Turton K., Chaddock J. A. and Acharya K. R. (2002) Botulinum and tetanus neurotoxins: structure, function and therapeutic utility. Trends Biochem. Sci. 27, 552-558.

Valls-Sole J., Tolosa E. S. and Ribera G. (1991) Neurophysiological observations on the effects of botulinum toxin treatment in patients with dystonic blepharospasm. J. Neurol. Neurosurg. Psychiatry 54, 310-313.

Verderio C., Rossetto O., Grumelli C., Frassoni C., Montecucco C. and Matteoli M. (2006) Entering neurons: botulinum toxins and synaptic vesicle recycling. EMBO Rep. 7, 995-999.

Verderio C., Grumelli C., Raiteri L., Coco S., Paluzzi S., Caccin P., Rossetto O., Bonanno G., Montecucco C. and Matteoli M. (2007) Traffic of botulinum toxins A and $\mathrm{E}$ in excitatory and inhibitory neurons. Traffic 8, 142-153.

Wang M. M., Janz R., Belizaire R., Frishman L. J. and Sherry D. M. (2003) Differential distribution and developmental expression of synaptic vesicle protein 2 isoforms in the mouse retina. J. Comp. Neurol. 460, 106-122. 
Wang J., Meng J., Lawrence G. W. et al. (2008) Novel chimeras of botulinum neurotoxins $\mathrm{A}$ and $\mathrm{E}$ unveil contributions from the binding, translocation, and protease domains to their functional characteristics. J. Biol. Chem. 283, 16993-17002.

Watson W. E. (1969) The response of rat hypoglossal neurones to injection of botulinum toxin into the tongue. J. Physiol. 202, 101P+.

Wiegand H. and Wellhoner H. H. (1977) The action of botulinum A neurotoxin on the inhibition by antidromic stimulation of the lumbar monosynaptic reflex. Naunyn Schmiedebergs Arch. Pharmacol. 298, 235-238.
Wiegand H., Erdmann G. and Wellhoner H. H. (1976) 125I-labelled botulinum A neurotoxin: pharmacokinetics in cats after intramuscular injection. Naunyn Schmiedebergs Arch. Pharmacol. 292, $161-165$.

Wohlfarth K., Schubert M., Rothe B., Elek J. and Dengler R. (2001) Remote F-wave changes after local botulinum toxin application. Clin. Neurophysiol. 112, 636-640.

Ziemann U., Hallett M. and Cohen L. G. (1998) Mechanisms of deafferentation-induced plasticity in human motor cortex. J. Neurosci. 18, 7000-7007. 